\title{
Aproximación al patrón alimentario de los inhumados en la cista de la Edad del Bronce de Ondarre (Aralar, Guipúzcoa) a través del análisis de isótopos estables de carbono y nitrógeno sobre colágeno óseo
}

\author{
Dietary reconstruction of the individuals interred in the Bronze Age burial cist of Ondarre \\ (Aralar, Guipuzcoa) through carbon and nitrogen stable isotope analysis on bone collagen
}

\author{
Teresa Fernández-Crespo ${ }^{a}$, Jose Antonio Mujikab ${ }^{b}$ Javier Ordoñob
}

\section{RESUMEN}

Se presentan los resultados del análisis de isótopos estables de carbono y nitrógeno sobre colágeno óseo de los individuos depositados en la cista guipuzcoana de la Edad del Bronce (II milenio cal. BC) de Ondarre. Los datos obtenidos sugieren una dieta mixta de origen terrestre basada en el consumo de plantas $\mathrm{C}_{3}$ y sobre todo de animales domésticos. No existen indicios de que los recursos marinos, lacustre-fluviales o las plantas $\mathrm{C}_{4}$ supusieran una contribución significativa a la subsistencia cotidiana, tal y como parece ocurrir en otros yacimientos coetáneos de la Península Ibérica y el archipiélago balear. La elevada ingesta proteica observada podría vincularse con el estatus de los inhumados y/o con la prevalencia de un modelo económico ganadero en que el consumo de productos animales tendría un gran peso. in other contemporary sites in Iberia and the Balearic islands, marine and freshwater resources and $C_{4}$ plants do not seem to have played an important role in everyday life subsistence. The observed high protein consumption may relate to the status of the people interred and/or to a dominant livestock economy where animal products would have a great importance in diet.

Palabras clave: Isótopos estables; Paleodieta; Restos humanos; Cista; Edad del Bronce; Aralar; País Vasco; Pirineo Occidental.

Key words: Stable isotopes; Paleodiet; Human remains; Cist burial; Bronze Age; Aralar; Basque Country; Western Pyrenees.

\section{INTRODUCCIÓN}

Los análisis de isótopos estables de carbono y nitrógeno sobre colágeno óseo gozan de una gran aceptación como vía de reconstrucción de la dieta de las comunidades pretéritas. Sin embargo, y aunque se llevan aplicando en la Arqueología europea desde los años 80 , el recurso a los mis-

\begin{abstract}
The results of carbon and nitrogen stable isotope analysis on bone collagen of the individuals interred in
the Bronze Age (II millennium cal. BC) cist burial of Ondarre suggest a mixed diet based on $C_{3}$ plants and
mainly domesticated terrestrial animals. As observed

ABSTRACT
\end{abstract}

\footnotetext{
a Dpto. de Genética, Antropología Física y Fisiología Animal. Universidad del País Vasco. B ${ }^{\circ}$ Sarriena. 48940 Leioa. España; Research Laboratory for Archaeology and the History of Art, University of Oxford. South Parks Road. OX1 3QY Oxford. United Kingdom. Correos e.: teresa.fernandezc@ehu.es; teresa.fernandez-crespo@rlaha.ox.ac.uk

b Dpto. de Geografía, Prehistoria y Arqueología, Universidad del País Vasco (UPV/EHU). C/ Tomás y Valiente s/n. 01006 Vitoria. España. Correos e.: joseantonio.mugica@ehu.es; javier.ordono@ehu.es

Recibido 26-I-2016; aceptado 16-III-2016.
} 
mos en la Península Ibérica ha sido prácticamente nulo hasta el lustro pasado. Los estudios centrados en la Edad del Bronce son muy contados (Arias 2005; Van Strydonck et al. 2005; Lillios et al. 2010; Nájera et al. 2010; McClure et al. 2011; Salazar-García y Vizcaíno 2011; García Borja et al. 2013; López-Costas et al. 2015). En su mayoría se ciñen a la fachada atlántica, la Submeseta sur, el Levante peninsular y el archipiélago balear, sin existir análisis de este tipo para estas cronologías en el área pirenaica occidental, donde se localiza la cista de Ondarre.

Estos estudios se fundamentan en que la composición de los tejidos humanos proviene de la ingesta alimentaria del individuo durante la última década de su vida (Ambrose y Norr 1993; Hedges et al. 2007). Durante el proceso de incorporación de los átomos de la dieta a los tejidos, conocido como fraccionamiento isotópico, la proporción entre el isótopo más pesado y el más ligero $\left({ }^{13} \mathrm{C} /{ }^{12} \mathrm{C}\right.$ o $\left.{ }^{15} \mathrm{~N} /{ }^{14} \mathrm{~N}\right)$ cambia, pero lo hace de una manera específica y conocida, lo que resulta esencial para poder identificar correctamente las principales fuentes de alimentación (Schoeller 1999). Los valores isotópicos resultantes de esta relación se representan mediante la notación delta $(\delta)$ en partes por mil (\%o), la cual indica su desviación con respecto a un estándar (carbono fósil marino, Vienna Pee Dee Belemnite VPDB; y nitrógeno atmosférico, atmospheric $N_{2}$ AIR).

En este contexto, los valores de los isótopos de carbono $\left(\delta^{13} \mathrm{C}\right)$ son conocidos por reflejar la proporción de alimentos de origen marino y terrestre consumidos, con resultados teóricamente cercanos al $-20 \pm 1 \%$ para dietas con un consumo proteico exclusivamente terrestre y en torno al $-12 \pm 1 \%$ en el caso marino (DeNiro y Epstein 1978; Richards et al. 2003). Asimismo, indican la presencia en la dieta de plantas con rutas fotosintéticas diferentes. Las plantas $\mathrm{C}_{3}$ de regiones templadas y frías como el trigo exhiben valores en torno al $-25 \%$ y las plantas $\mathrm{C}_{4}$ de regiones tropicales, áridas o semiáridas como el mijo en torno al $-12 \%$ (O'Leary 1988). Los valores de los isótopos de nitrógeno $\left(\delta^{15} \mathrm{~N}\right)$, por su parte, representan la ratio entre productos de génesis animal y vegetal, esto es, su posición en la cadena trófica, mostrando un incremento del 3-5\% por cada peldaño que asciende el individuo (Schoeninger et al. 1983; Minagawa y Wada 1984; Post 2002; Bocherens y Drucker 2003), si bien la distinción entre dietas cárnicas o principalmente vegetarianas parece menos sencilla de lo que originalmente se creía (Hedges y Reynard 2007; Warinner et al. 2013).

Partiendo de estas premisas, el presente estudio pretende profundizar en la dieta y en los modos de subsistencia de los individuos inhumados en la cista de Ondarre a través del estudio de los isótopos estables de carbono y nitrógeno sobre colágeno óseo.

\section{LA CISTA DE ONDARRE}

La cista de Ondarre se sitúa en el sector centro-occidental de la Sierra de Aralar (Guipúzcoa), concretamente en la base del cono que desciende del entorno de Esnaurreta-Intzensaro, a una altitud de 760 m.s.n.m (Fig. 1). El emplazamiento presenta profundas incisiones de origen erosivo provocadas por las aguas que descendían por la ladera contigua y que iban a parar a la sima de Ondarreko Zuloa. Estas cárcavas delimitan interfluvios a modo de montículos o islotes más o menos amplios. En el borde de uno de ellos, y en el extremo más próximo a la sima, se construyó el monumento funerario.

El paraje, hoy en día dominado por herbáceas, estuvo poblado durante los inicios de la Edad de Bronce por bosques caducifolios de avellanos, robles, encinas y alisos, con escasos arbustos (brezos y rosáceas espinosas) que en todo caso ocuparían espacios abiertos en los claros de los bosques junto con algunas praderas de gramíneas y pastizales de uso ganadero. Sin embargo, no se han documentado por el momento indicadores de la existencia de prácticas agrícolas ni aquí ni en el vecino yacimiento de habitación coetáneo de Esnaurreta (Pérez-Díaz com. pers.), ni en otras cavidades próximas como Iritegi (Moreno-Larrazabal et al. 2014). A este respecto, algunos autores consideran que la habitual localización de cistas y otras pequeñas estructuras megalíticas a una notable altitud durante la Edad de Bronce puede interpretarse como resultado de la explotación de estas tierras altas en movimientos estivales de más o menos largo recorrido (Mujika y Edeso 2012). Esta práctica se evidencia en diferentes épocas históricas (Agirre et al. 2003-2007; Mujika et al. 2013) y pervive todavía hoy en la zona.

Trab. Prehist., 73, N. ${ }^{\circ}$ 2, julio-diciembre 2016, pp. 325-334, ISSN: 0082-5638

doi: $10.3989 /$ tp.2016.12177 


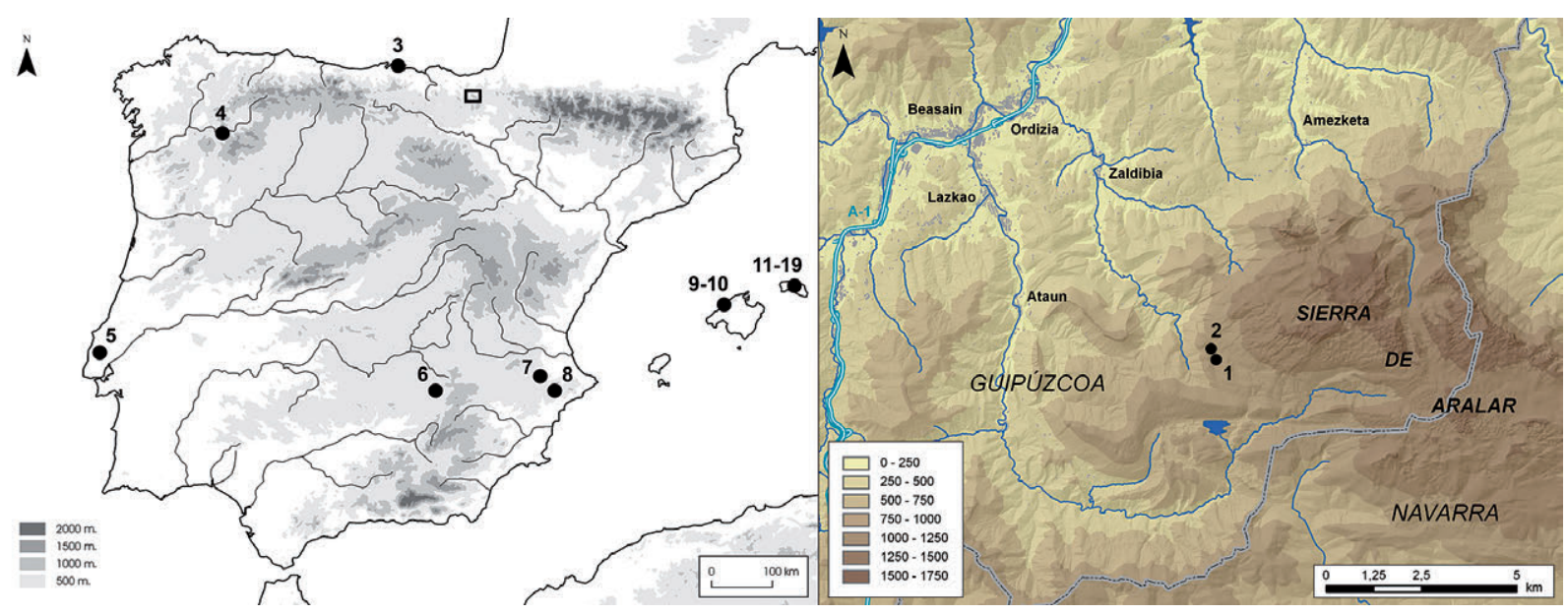

Fig. 1. Localización de la cista de Ondarre (Aralar, Guipúzcoa) (1), del cercano yacimiento de Esnaurreta II (2) y de otros yacimientos contemporáneos de la Península Ibérica y las Islas Baleares con análisis isotópicos de carbono y nitrógeno mencionados en el texto: La Garma (3); Cova do Santo (4); Bolores (5); Motilla del Azuer (6); Coveta del Frare (7); Cova de la Pastora (8); Cova Gregoria B (9); Son Gallard (10); Alcaïdus (11); Biniai Nou (12); Cala Morell (13); Càrritx (14); Es Tudons (15); Rafal Rubí (16); Ses Arenes (17); Ses Aritges (18); Ses Roques Llises (19). Fuentes cartográficas: hojas digitales 88, 89, 113 y 114 del MTN25 vectorial (eds. 2003-2008) y MDT25 (ed. 2010) del Instituto Geográfico Nacional de España.

La excavación, dirigida por uno de los firmantes (J. A. Mujika) en 2011, identificó una pequeña cámara de $150 \mathrm{~cm}$ de longitud por $90-110 \mathrm{~cm}$ de anchura y 50-60 cm de altura, conformada al menos por ocho losas de caliza y una de arenisca muy alteradas físico-químicamente. Estaban desplazadas de su posición original, observándose una clara basculación de las losas verticales y el desplome de la cubierta (Fig. 2). Los trabajos de campo recuperaron diversos huesos humanos junto con pequeños fragmentos cerámicos que corresponden a 7 recipientes sin decorar (dos cuencos, un vaso carenado y un recipiente de perfil en S), 1 posible colgante muy deteriorado sobre una gruesa lámina ósea fracturada con cinco pequeños puntos decorativos, 1 cristal de cuarzo bipiramidal y 18 objetos de sílex (quince restos de talla, un fragmento de punta de dorso sin ápice, procedente posiblemente de una fugaz ocupación anterior del lugar, una pieza astillada y un fragmento de raedera lateral de talón diedro). Bajo estos restos, se definió un área de combustión (90 cm de longitud y $40 \mathrm{~cm}$ de anchura) que se extendía por la mitad norte del recinto. Ésta ha sido interpretada como resultado de un pequeño fuego de carácter ritual relacionado con la construcción del monumento, $\mathrm{y}$ se ha fechado a partir de carbones en 3880 \pm 30 BP (2467-2236 cal. BC, $2 \sigma$; Beta-413177) y 3730 \pm 30 BP (2266-2032 cal. BC, 2o; Beta-327177). Además, con el fin de acotar su reutilización se dataron dos individuos, uno adulto y otro infantil, depositados en el mismo. El primero ofreció una fecha de $3260 \pm 30$ BP (1616-1454 cal. BC, 2б; Beta-350136) y el segundo una de $2620 \pm 30$ BP (831-775 cal. BC, 20; Beta-327176).

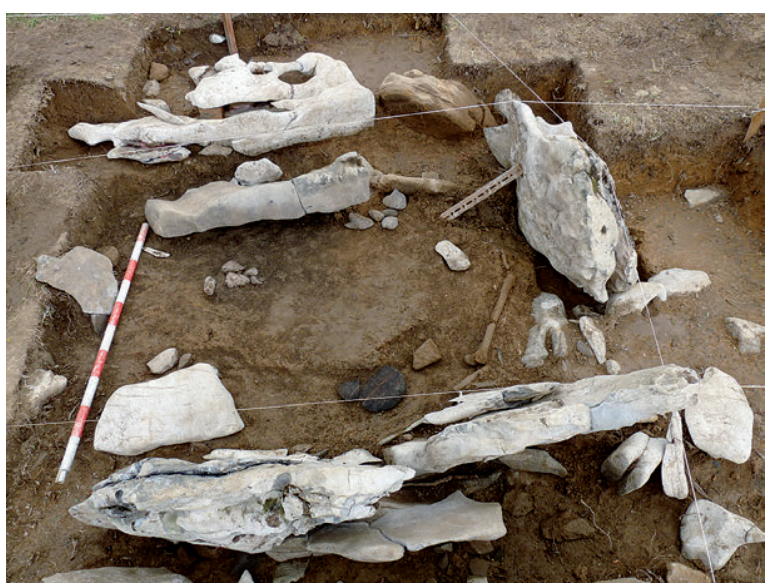

Fig. 2. Vista de la cámara de la cista de Ondarre desde el lado meridional (foto J. A. Mujika 2011). 


\section{LOS RESTOS HUMANOS}

El material óseo humano recuperado de la cista de Ondarre es escaso. Su estado de conservación es muy deficiente, resultado de diversos procesos postdeposicionales que han fragmentado y alterado de manera importante la estructura de los huesos.

La colección se compone de 3 fragmentos de cráneo adulto, 1 fragmento de mandíbula adulto con un canino y dos premolares in situ, 2 primeras y 3 segundas vértebras cervicales (una subadulta), 15 fragmentos vertebrales indeterminados, 4 fragmentos de costillas indeterminados, parte de una escápula adulta, 5 fragmentos de húmero (dos subadultos), 7 fragmentos de cúbito (dos subadultos), 7 de radio (dos subadultos), 11 falanges de mano (tres subadultas), 6 metacarpianos (uno subadulto), 2 pisiformes (uno subadulto), 2 semilunares adultos, 4 fragmentos de coxal adultos, 1 fragmento de fémur adulto, 4 rótulas (una subadulta), 3 fragmentos de tibia (uno subadulto), 3 fragmentos de peroné (uno subadulto), 2 falanges de pie adultas, 9 metatarsianos (uno subadulto), 1 navicular adulto, parte de 2 calcáneos adultos y 2 astrágalos adultos, 30 pequeños fragmentos diafisarios de huesos largos indeterminados y 659 esquirlas óseas. Además se han contabilizado un total de 72 piezas dentales sueltas (Tab. 1).

Las piezas deciduas y los gérmenes dentarios de incisivos, caninos y molares en distinto estadio de formación, así como los múltiples vestigios esqueléticos inmaduros, son consistentes con la presencia de, al menos, dos sujetos subadultos (Stloukal y Hanáková 1978; Ubelaker 1989; Scheuer y Black 2000). Del mismo modo, el recuento de las piezas dentales permanentes y su distinto grado de atrición (Brothwell 1965), y la repetición de fragmentos del esqueleto apendicular (por ejemplo las rótulas) dan fe de la exis-

\begin{tabular}{|c|c|c|c|c|c|c|}
\hline Pieza & Incisivo & Canino & Premolar & Molar & Indet. & Total \\
\hline Decidua & 3 & 7 & - & 4 & 3 & 17 \\
\hline Permanente & 10 & 7 & 9 & 12 & 7 & 45 \\
\hline Total & 13 & 14 & 9 & 16 & 10 & 62 \\
\hline
\end{tabular}

Tab. 1. Relación de piezas dentales de los inhumados en la cista de la Edad del Bronce de Ondarre (Aralar, Guipúzcoa). tencia de, al menos, dos sujetos adultos (White y Folkens 2005: 339).

El número mínimo de individuos o NMI se ha estimado en 4, caracterizados como un infantil de $18 \pm 6$ meses, un niño de $7 \pm 2$ años, un adulto joven de unos 20-30 años (visto el leve desgaste dental de algunos dientes permanentes y la ausencia de cambios degenerativos a nivel postcraneal) y un adulto probablemente maduro de unos 40-50 años (dada la importante atrición de ciertas piezas dentarias y las artropatías detectadas en diversos huesos).

Como datos paleopatológicos, referimos únicamente la existencia de una pequeña caries cervical y otra oclusal en sendos premolares definitivos, y leves signos artrósicos en una falange, una rótula, varios metatarsianos, dos calcáneos y dos astrágalos adultos. También reseñamos varias bandas hipoplásicas en un canino superior derecho, generalmente asociadas a un episodio de estrés biológico durante la infancia (destete, enfermedad...).

\section{MATERIAL}

De cara al estudio isotópico de los inhumados en Ondarre se han seleccionado tres muestras óseas procedentes de rótulas, pertenecientes a un sujeto subadulto de unos siete años de edad (OND3) y dos adultos de sexo indeterminado, uno joven y otro maduro (OND4 y OND2, respectivamente). La selección de este hueso responde a su relativamente correcta representatividad con respecto al NMI (recordemos, estimado en cuatro), nada baladí dadas las graves penurias de conservación de la colección. Así, solo se ha excluido del muestreo un infantil de $18 \pm 6$ meses, cuyos valores isotópicos estarían por otra parte presumiblemente determinados por la señal de la lactancia materna. Ante la ausencia de fauna con que establecer los parámetros medios del ecosistema local y comparar las muestras humanas, se tomó una muestra de costilla de ovicáprido de Esnaurreta II, un yacimiento de habitación contemporáneo (datado en el primer tercio del II milenio cal. BC) y muy próximo a Ondarre (a tan solo $300 \mathrm{~m}$ lineales), a fin de disponer de material faunístico válido (Mays 2010). El peso de todas las muestras tomadas ha oscilado entre los 500 y los $800 \mathrm{mg}$.

Trab. Prehist., 73, N. ${ }^{\circ}$ 2, julio-diciembre 2016, pp. 325-334, ISSN: 0082-5638

doi: $10.3989 /$ tp.2016.12177 


\section{MÉTODOS}

Las mediciones de los valores de los isótopos estables de carbono y nitrógeno se han llevado a cabo en el Research Laboratory for Archaeology and the History of Art (RLAHA) de la Universidad de Oxford, donde se prepararon y analizaron todas las muestras. El protocolo de extracción del colágeno utilizado ha sido el método de Longin (1971), modificado y descrito por Richards y Hedges (1999). El proceso puede resumirse en diez pasos principales: 1) limpieza de las muestras mediante abrasión con polvo de óxido de aluminio, 2) triturado de las muestras, 3) desmineralización en $0,5 \mathrm{M} \mathrm{HCl}$ a $\left.4^{\circ} \mathrm{C}, 4\right)$ drenado de la solución ácida y enjuague en agua destilada, 5) gelatinización en $\mathrm{pH} 3$ aproximadamente a $75^{\circ} \mathrm{C}$ durante 48 horas, 6) filtrado de la solución con malla de 5-8 $\mu \mathrm{m}, 7$ ) congelación a $-40^{\circ} \mathrm{C}$ durante al menos 4 horas, 8) liofilización, 9) pesado e introducción del colágeno resultante en pequeñas cápsulas de aluminio, y 10) combustión y análisis de éstas en un espectrómetro de masas (en este caso, un SERCON 20-22 acoplado a un analizador elemental).

Todas las muestras se analizaron por duplicado, situándose la precisión analítica tanto para $\delta^{13} \mathrm{C}$ como para $\delta^{15} \mathrm{~N}$ dentro de un intervalo inferior al $\pm 0,2 \%$ o $(1 \sigma)$. También se han analizado estándares de valores isotópicos conocidos (alanina, foca marina, cafeína) con el objetivo de calibrar el funcionamiento de la máquina y comprobar la validez de los datos. Finalmente, la calidad bioquímica del colágeno se ha comprobado utilizando los criterios más comunes: $\% \mathrm{Col}(>1), \% \mathrm{C}$ $(>30), \% \mathrm{~N}(>11)$ y C:N $(2,9-3,6)$ (Ambrose 1993; DeNiro 1985; Van Klinken 1999).

\section{RESULTADOS}

Se ha podido extraer colágeno de las cuatro muestras tomadas. Éstas presentan buenos índices de preservación $(\% \mathrm{Col}, \% \mathrm{C}, \% \mathrm{~N}, \mathrm{C}: \mathrm{N})$ salvo en el caso de OND3, cuyos porcentajes de carbono y nitrógeno y el rendimiento del colágeno son inferiores a los valores estipulados (Tab. 2). No obstante, como ofrece un $\mathrm{C}: \mathrm{N}$ aceptable y los valores isotópicos son congruentes con las otras muestras, se ha incluido en el análisis y discusión.

Las muestras humanas analizadas han arrojado un valor $\delta^{13} \mathrm{C}$ medio de $-21,3 \pm 0,4 \%$ o $(1 \sigma)$, y sus valores mínimos y máximos son $-21,6$ y $-20,9 \%$. $\mathrm{Su}$ valor medio $\delta^{15} \mathrm{~N}$ es de $9,4 \pm 0,6 \%$ o $(1 \sigma)$, y sus valores mínimos y máximos son 8,9 y 10,0\%. La diferencia entre estos valores medios y los del ovicáprido de Esnaurreta II es de 1,1 y 5,4\%o para $\delta^{13} \mathrm{C}$ y $\delta^{15} \mathrm{~N}$, respectivamente.

\section{DISCUSIÓN}

Las tres muestras humanas de Ondarre analizadas dan fe de valores de carbono y nitrógeno relativamente homogéneos pero que dejan lugar a cierta variabilidad. A este respecto, el niño no muestra diferencias reseñables con relación a los adultos, situándose sus valores muy cerca de los del adulto joven. El ligero empobrecimiento especialmente en el nitrógeno de este sujeto inmaduro podría atribuirse a procesos fisiológicos relacionados con el crecimiento, una dieta diferente $o$, más posiblemente, una combinación de ambos (Beaumont et al. 2013). El adulto maduro ostenta claramente valores isotópicos más elevados. No

\begin{tabular}{|c|c|c|c|c|c|c|c|c|c|c|}
\hline Yacimiento & Ref. & Especie & Edad & Hueso & $\mathbf{\%} \mathbf{C o l}$ & $\mathbf{\%} \mathbf{C}$ & $\mathbf{\% N}$ & $\mathbf{C : N}$ & $\delta^{13} \mathbf{C}(\% \mathbf{0})$ & $\delta^{\mathbf{1 5}} \mathbf{N}(\% \mathbf{0})$ \\
\hline Ondarre & OND2 & Humano & Adulto & Rótula dcha. & 4,7 & 35,9 & 12,5 & 3,4 & $-20,9$ & 10,0 \\
\hline Ondarre & OND3 & Humano & Subadulto & Rótula izq. & 0,6 & 26,8 & 8,9 & 3,5 & $-21,6$ & 8,9 \\
\hline Ondarre & OND4 & Humano & Adulto & Rótula dcha. & 6,3 & 44,8 & 15,8 & 3,3 & $-21,5$ & 9,2 \\
\hline Esnaurreta II & ESN5 & $\begin{array}{c}\text { Ovis aries/Capra } \\
\text { hircus }\end{array}$ & Indet. & Costilla & 1,3 & 33,3 & 11,6 & 3,4 & $-22,2$ & 4,3 \\
\hline
\end{tabular}

Tab. 2. Resultados de los análisis de isótopos de carbono y nitrógeno para las muestras de Ondarre y Esnaurreta II, con referencia a los índices de preservación del colágeno y a la caracterización arqueoantropológica de cada resto. Nótese que los índices de OND3 mostrados en cursiva muestran valores ligeramente por debajo de lo aceptable.

Trab. Prehist., 73, N. ${ }^{\circ} 2$, julio-diciembre 2016, pp. 325-334, ISSN: 0082-5638 doi: $10.3989 /$ tp.2016.12177 
obstante, desconocemos si este enriquecimiento podría relacionarse con su edad, sexo, estatus $\mathrm{y} / \mathrm{u}$ otras variables como por ejemplo la cronología.

La falta de una muestra robusta de fauna con que establecer los parámetros medios del ecosistema local limita sin duda la interpretación de la dieta de los humanos inhumados en la cista de Ondarre. Asumiendo el incremento del 3-5\% en el $\delta^{15} \mathrm{~N}$ típicamente aceptado por cada peldaño trófico (Schoeninger et al. 1983; Minagawa y Wada 1984; Post 2002; Bocherens y Drucker 2003), los resultados sugieren una dieta mixta basada en plantas $\mathrm{C}_{3} \mathrm{y}$ muy especialmente en productos animales eminentemente terrestres, marcada por un elevado consumo de proteínas. Esta importante ingesta proteica, comúnmente ligada al estatus en la literatura arqueológica (Lillie 1997; Danforth 1999), no casaría mal con el tradicional prestigio atribuido a los inhumados en cistas (García Sanjuán et al. 1999) y/o con un posible modelo de subsistencia basado principalmente en la ganadería (Mujika y Edeso 2012) con un consumo notable de carne y productos secundarios. Desafortunadamente, apenas se dispone de datos que refuercen una $u$ otra hipótesis. La naturaleza de las estructuras funerarias y de los ajuares encontrados, siendo el elemento más frecuente la cerámica, no parece a priori muy indicativa de la posición social de los inhumados. A su vez el parco número de yacimientos de habitación de esta cronología identificados y excavados por el momento en el Pirineo occidental y el prácticamente nulo registro zooarqueológico disponible de asentamientos en zonas de montaña impiden precisar las características y peculiaridades del modelo económico. Los contados y pobres asentamientos conocidos cuentan con las especies clásicas (vaca, oveja/cabra y cerdo), sin detectarse composiciones ganaderas peculiares que permitieran sospechar la existencia de rebaños específicos (Edeso et al. 2010).

Además hay que reconocer graves limitaciones a la hora de discernir el peso real de las plantas en la dieta, ya que los resultados isotópicos reflejan sobre todo la ingesta de proteínas que los vegetales poseen en poca cantidad, lo que enmascara su consumo (Ambrose y Norr 1993; Tieszen y Fagre 1993; Jim et al. 2006). Asimismo, los valores medios de cereales, leguminosas y otros vegetales que podrían haber formado parte de la dieta no han podido ser adecuadamente establecidos, por la práctica ausencia de macrorrestos analizables. Sin embargo, algunos estudios de antracología sugieren la interesante idea de que la recolección de frutos silvestres, especialmente bellotas y avellanas, pudo jugar un papel destacado en la subsistencia de las poblaciones de la región (Moreno-Larrazabal et al. 2014). Además, es bien sabido que las poblaciones montanas tradicionales asumían muy bajos rendimientos agrarios, fundamentalmente cerealísticos, como única alternativa posible a un transporte difícil y muy costoso desde áreas mejor dotadas para la explotación ${ }^{1}$.

Otro factor a considerar es el desconocimiento del impacto que pudieron haber tenido ciertas prácticas económicas y culturales como el uso de abono, el consumo de productos no locales o el empleo de diferentes forrajes para alimentar a distintas especies animales. Sin ir más lejos, el uso de fertilizantes naturales produciría un enriquecimiento en los valores de ${ }^{15} \mathrm{~N}$ del ganado (Fraser et al. 2013), mientras la inclusión de plantas $\mathrm{C}_{4}$ en los forrajes tendría la misma consecuencia en el ${ }^{13} \mathrm{C}$ (Zavodny et al. 2014). Estos cambios se reflejarían evidentemente en los valores $\delta^{15} \mathrm{~N}$ y $\delta^{13} \mathrm{C}$ de la cabaña ganadera pero también en cierto modo en la señal isotópica de las personas que se alimentasen de estos animales o de productos derivados de los mismos.

Igual de complicado es valorar la contribución de los peces de río a esta dieta, ya que su composición isotópica suele ser muy variable (Fuller et al. 2012). Sin embargo, podemos afirmar que, en comparación con el único herbívoro disponible, la huella isotópica de los inhumados en Ondarre no muestra indicios de que las proteínas de agua dulce supusieran una contribución significativa a la subsistencia cotidiana, como tampoco parecen haberlo hecho los recursos de origen marino a tenor de los resultados obtenidos $\left({ }^{13} \mathrm{C}\right.$ empobrecido). Esto no implica que nunca se comieran sino que su consumo, de darse, habría sido realmente eventual $\mathrm{y}$, en cualquier caso, no pudo suponer más del $5-15 \%$ de las proteínas ingeridas, sin haber dejado una señal isotópica clara (Richards y Schulting 2006).

Los estudios de isótopos estables de carbono y nitrógeno sobre colágeno óseo para poblaciones

1 López de Calle, C. 1993: Los sepulcros megalíticos de Cameros (La Rioja). Tesis doctoral inédita. Universidad de Zaragoza. Zaragoza.

Trab. Prehist., 73, N. ${ }^{\circ}$ 2, julio-diciembre 2016, pp. 325-334, ISSN: 0082-5638

doi: $10.3989 /$ tp.2016.12177 
del II milenio cal. BC de la Península Ibérica y el archipiélago balear son relativamente escasos y corresponden en su amplia mayoría a enterramientos en cavidades. En general, como en Ondarre, los datos isotópicos del periodo apuntan a una alimentación mixta basada en esencia en plantas cultivadas y complementada con el consumo de animales domésticos y, quizá en menor medida, salvajes (Fig. 3). Sin embargo, entrando en detalle, también se observa una variabilidad geográfica en los valores de ${ }^{13} \mathrm{C}$, muy posiblemente influenciada por las condiciones climáticas de cada región, con valores más empobrecidos en la fachada atlántica, sobre todo en la cornisa cantábrica, y más enriquecidos en el interior peninsular, mientras la región mediterránea presenta ratios intermedios. También existe cierta diversidad en el ${ }^{15} \mathrm{~N}$, pero en este caso sin un patrón claro, algo esperable ante la pluralidad de fuentes de variación isotópica que pueden explicar dicha heterogeneidad: nivel de aridez, aportes proteicos, prácticas económico-culturales (por ejemplo, el uso de abonos), estrés biológico.

En la fachada atlántica, los valores medios más parecidos a los aquí presentados son los de los inhumados en la cavidad orensana de Cova do Santo (x

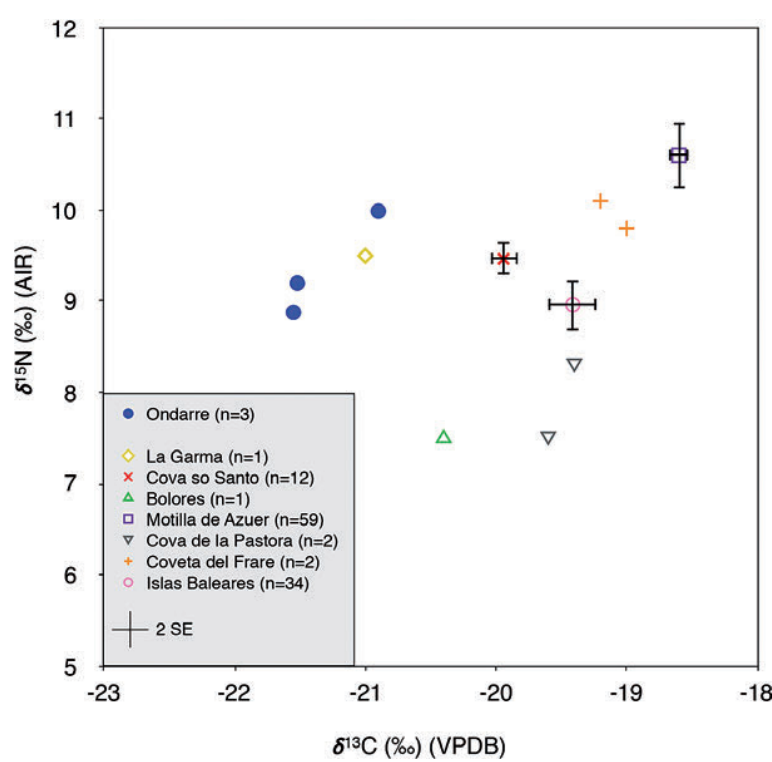

Fig.3. Diagrama de puntos en que se incluyen los valores humanos de $\delta^{13} \mathrm{C}$ y $\delta^{15} \mathrm{~N}$ de Ondarre y de otros yacimientos del II milenio cal. BC de la Península Ibérica e Islas Baleares con estudio isotópico, a modo de comparación. $\mathrm{SE}=$ error típico o estándar de la media.

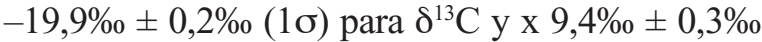
$(1 \sigma)$ para $\delta^{15} \mathrm{~N} ; \mathrm{n}=13$ ) (López-Costas et al. 2015) $\mathrm{y}$, especialmente, los del esqueleto encontrado en la cueva cántabra de La Garma $\left(-21,0 \%\right.$ para $\delta^{13} \mathrm{C}$ y 9,5\% para $\delta^{15} \mathrm{~N}$ ) (Arias 2005). Éstos evidencian que, a pesar de la proximidad al mar del segundo, en su dieta no hay un aporte marino mensurable. En la región lusa, un niño hallado en la cavidad funeraria lisboeta de Bolores $\left(-20,4 \%\right.$ para $\delta^{13} \mathrm{C}$ y 7,5\%o para $\delta^{15} \mathrm{~N}$ ) (Lillios et al. 2010) muestra una dieta similar, con proteínas procedentes sobre todo de plantas $\mathrm{C}_{3}$. En cambio, en el interior peninsular, el asentamiento ciudadrealeño de la Motilla del Azuer $(\mathrm{x}-18,6 \%$ o, $\pm 3 \%$ ( $1 \sigma)$ para $\delta^{13} \mathrm{C}$ y x $10,6 \%$ o $\pm 0,3 \%$ o $(1 \sigma)$ para $\left.\delta^{15} \mathrm{~N} ; \mathrm{n}=59\right)$ (Nájera et al. 2010) exhibe un ligero enriquecimiento en los valores de carbono. En origen se atribuyó al consumo de recursos marinos, a pesar de su considerable distancia al mar $(200 \mathrm{~km})$, para después achacarse a la posible introducción de cereales $\mathrm{C}_{4}$, en concreto mijo, en la dieta (López-Costas et al. 2015). En nuestra opinión, la aridez constatada en el registro paleoambiental (Sánchez 2014) debería ser igualmente un factor a valorar. En el litoral mediterráneo, los valores de los dos sujetos de la Edad del Bronce inhumados en la oquedad alicantina de Cova de la Pastora (x $-19,5 \%$ $\pm 0,1 \%$ o $(1 \sigma)$ para $\delta^{13} \mathrm{C}$ y x $7,9 \%$ $\pm 0,6 \%$ o $(1 \sigma)$ para $\delta^{15} \mathrm{~N}$; $\mathrm{n}=2$ ) (McClure et al. 2011) y de sendos individuos exhumados del yacimiento valenciano de la Coveta del Frare $(\mathrm{x}-19,1 \%$ $\pm 0,1 \%$ o $(1 \sigma)$ para $\delta^{13} \mathrm{C}$ y x $10,0 \%$ o $\pm 0,2 \%$ o $(1 \sigma)$ para $\left.\delta^{15} \mathrm{~N} ; \mathrm{n}=2\right)$ (García Borja et al. 2013) son nuevamente consistentes con una dieta basada en productos terrestres $\mathrm{C}_{3}$ en la que no se aprecia ingesta de proteínas de origen marino ni lacustre-fluvial. Finalmente, para las Islas Baleares los resultados dan fe de un patrón alimentario semejante. Así, el análisis conjunto de los valores obtenidos en individuos de esta cronología de los contextos funerarios de Alcaïdus, Biniai Nou, Cala Morell, Càrritx, Cova Gregoria B, Es Tudons, Rafal Rubí, Ses Arenes, Ses Aritges, Ses Roques Llises y Son Gallard (x $-19,4 \%$ $\pm 0,5 \%$ ( $1 \sigma)$ para $\delta^{13} \mathrm{C}$ y x $9,0 \%$ $\pm 0,8 \%$ o $(1 \sigma)$ para $\left.\delta^{15} \mathrm{~N} ; \mathrm{n}=34\right)$ (Van Strydonck et al. 2005) demuestra que, salvo contadas excepciones, ni siquiera en contextos insulares se observa un uso significativo de productos del mar, constituyendo un patrón alimentario semejante al identificado en otras regiones de Europa durante el mismo período (Montgomery y Jay 2013). 


\section{CONCLUSIONES}

Los resultados de isótopos estables de carbono y nitrógeno sobre colágeno óseo de la cista de Ondarre sugieren una dieta mixta de origen terrestre basada en el consumo de plantas $\mathrm{C}_{3} \mathrm{y}$ de animales domésticos, sin indicios de que los recursos marinos, lacustre-fluviales o las plantas $\mathrm{C}_{4}$ supusieran una contribución significativa a la subsistencia cotidiana. La elevada ingesta proteica atestiguada podría vincularse con el estatus de los inhumados, siendo las cistas supuestamente concebidas para inhumar personajes de cierto prestigio social, o incluso con un modelo de subsistencia ganadero centrado sobre todo en el consumo de productos animales. Sin embargo, un mejor conocimiento de los lugares de habitación, de los parámetros medios del ecosistema local (flora y fauna arqueológicas) y más estudios isotópicos sobre sujetos depositados en otras cistas del entorno serían necesarios para contrastar estas hipótesis. Los resultados son además perfectamente coherentes con los obtenidos en otros yacimientos de la Edad del Bronce en la Península Ibérica y el archipiélago balear, y especialmente semejantes a los procedentes de la fachada atlántica.

\section{AGRADECIMIENTOS}

Al Ministerio de Ciencia e Innovación(HAR201126956), al Gobierno Vasco (POS_2013_1_147; POS_2014_2_24; POS_2015_2_0001) y a la Universidad del País Vasco (IT-542-10/GIC10/46; IT-622-13), la financiación que ha permitido desarrollar esta investigación. A los evaluadores de la revista, por las sugerencias y comentarios que han contribuido a mejorar el trabajo.

\section{BIBLIOGRAFÍA}

Agirre, J.; Moraza, A.; Mujika, J. A.; Reparaz, X. y Telleria, E. 2003-07: "Primeros vestigios de un modelo económico de ganadería estacional especializada. Los fondos de cabaña tumulares de Arrubi y Esnaurreta (Aralar)". Kobie XXVII: 105-131.

Ambrose, S. H. 1993: "Isotope analysis of paleodiets: methodological and interpretive considerations".
En M. K. Sandford (ed.): Investigations of ancient human tissue: chemical analyses in Anthropology. Gordon and Breach. Pennsylvania: 59-130.

Ambrose, S. H. y Norr, L. 1993: "Experimental evidence for the relationship of the carbon isotope ratios of whole diet and dietary protein to those of bone collagen and carbonate". En J. B. Lamber y G. Grupe (eds.): Prehistoric human bone. Archaeology at the molecular level. Springer-Verlag. Berlín: 1-37.

Arias, P. 2005: "Determinaciones de isótopos estables en restos humanos de la región cantábrica. Aportación al estudio de la dieta de las poblaciones del Mesolítico y el Neolítico". Munibe (AntropologiaArkeologia) 57: 359-374.

Beaumont, J., Gledhill, A.; Lee-Thorp, J. y Montgomery, J. 2013: "Childhood diet: a closer examination of the evidence from dental tissues using stable isotope analysis of incremental human dentine". Archaeometry 55 (2): 277-295.

Bocherens, H. y Drucker, D. G. 2003: "Trophic level isotopic enrichments for carbon and nitrogen in collagen: case studies from recent and ancient terrestrial ecosystems". International Journal of Osteoarchaeology 13 (1-2): 46-53.

Brothwell, D. R. 1965: Digging up bones. The excavation, treatment and study of human skeletal remains. Cornell University Press. Ithaca.

Danforth, M. E. 1999: "Nutrition and politics in Prehistory". Annual Review of Anthropology 28: 1-25.

DeNiro, M. 1985: "Postmortem preservation and alteration of in vivo bone collagen isotope ratios in relation to palaeodietary reconstruction". Nature 317: 806-809.

DeNiro, M. J. y Epstein, S. 1981: "Influence of diet on the distribution of nitrogen isotopes in animals". Geochimica et Cosmochimica Acta 45: 341-351.

Edeso, J. M.; Mendizabal, M. y Mujika, J. A. 2010: "Estrategias de gestión de los recursos de montaña por las poblaciones dolménicas y otros grupos humanos contemporáneos en el Pirineo Occidental". En J. Fernández Eraso y J. A. Mujika (eds.): Congreso Internacional sobre el megalitismo y otras manifestaciones funerarias contemporáneas en su contexto social, económico y cultural (Beasain-Idiazabal 2007). Munibe suplemento 32, Universidad del País Vasco (UPV/EHU), Sociedad de Ciencias Aranzadi. Donostia-San Sebastián: 368-390.

Fraser, R. A.; Bogaard, A.; Schäferb, M.; Arbogastc, R. y Heaton, T. H. E. 2013: "Integrating botanical, faunal and human stable carbon and nitrogen isotope values to reconstruct land use and palaeodiet at LBK Vaihingen an der Enz, Baden-Württemberg”. World Archaeology 45: 492-517.

Fuller, B. T.; Müldner, G.; Van Neer, W.; Ervynck, A. y Richards, M. P. 2012: "Carbon and nitrogen stable isotope ratio analysis of freshwater, brackish and marine fish from Belgian archaeological sites 
( $1^{\text {st }}$ and $2^{\text {nd }}$ millennium AD)". Journal of Analytical Atomic Spectrometry 27: 807-820.

García Borja, P.; Pérez, A.; Biosca, V.; Ribera, A. y Salazar-García, D. C. 2013: "Los restos humanos de la Coveta del Frare (La Font de la Figuera, Valencia)". En P. García Borja, E. Revert, A. Ribera y V. Biosca (eds.): El naixement d'un poble. Història $i$ arqueologia de La Font de la Figuera. Ajuntament de la Font de la Figuera, Diputació Provincial de València. Valencia: 47-59.

García Sanjuán, L.; Hunt, M.; Hurtado, V.; Mondéjar, P. y Romero, E. 1999: "La ocupación humana en la comarca de la Sierra durante la Edad del Bronce". En Actas XII Jornadas del Patrimonio de la Comarca de la Sierra (Aracena, Huelva 1997): 149-180. Aracena.

Hedges, R. E. M.; Clement, J. G.; Thomas, D. L. y O'Connell, T. C. 2007: "Collagen turnover in the adult femoral mid-shaft: modelled from anthropogenic radiocarbon tracer measurements". American Journal of Physical Anthropology 133: 808-816.

Hedges, R. y Reynard, L. 2007: "Nitrogen isotopes and the trophic level of humans in archaeology". Jounal of Archaeological Science 34: 1240-1251.

Jim, S.; Jones, V.; Ambrose, S. H. y Evershed, R. P. 2006: "Quantifying dietary macronutrient sources of carbon for bone collagen biosynthesis using natural abundance stable carbon isotope analysis". British Journal of Nutrition 95: 1055-1062.

Lillie, M. 1997: "Women and children in Prehistory: resource sharing and social stratification at the Mesolithic-Neolithic transition in Ukraine". En J. Moore y E. C. Scott (eds.): Invisible and processes: writing gender and chilhood into European Archaeology. Leicester University Press. London: 213-228.

Lillios, K. T.; Waterman, A. J.; Artz, J. A. y Josephs, R. L. 2010: "The Neolithic-Early Bronze Age mortuary rockshelter of Bolores, Torres Vedras, Portugal". Journal of Field Archaeology 35: 19-39.

Longin, R. 1971: "New method of collagen extraction for radiocarbon dating". Nature 230: 241-242.

López-Costas, O; Müldner, G. y Martínez, A. 2015: "Diet and lifestyle in Bronze Age Northwest Spain: the collective burial of Cova do Santo". Journal of Archaeological Science 55: 209-218.

Mays, S. 2010: The archaeology of human bones. Routledge. London.

McClure, S. B.; García, O.; Roca de Togores, C.; Culleton, B. J. y Kennett, D. J. 2011: “Osteological and paleodietary investigation of burials from Cova de la Pastora, Alicante, Spain". Journal of Archaeological Science 38: 420-428.

Minagawa, M. y Wada, E. 1984: "Stepwise enrichment of ${ }^{15} \mathrm{~N}$ along food chains: further evidence and the relation between ${ }^{15} \mathrm{~N}$ and animal age". Geochimica et Cosmochimica Acta 48 (5): 1135-1140.
Montgomery, J. y Jay, M. 2013: "The contribution of skeletal isotope analysis to understanding the Bronze Age in Europe". En H. Fokkens y A. Harding (eds.): The Oxford handbook of the European Bronze Age. Oxford University Press. Oxford: 179-196.

Moreno-Larrazabal, A.; Uribarri, E.; Peñalver, X. y Zapata, L. 2014: "Fuelwood, crops and acorns from Iritegi cave (Oñati, Basque Country)". Environmental Archaeology 19 (2): 166-175.

Mujika, J. A.; Agirre, J.; Edeso, J. M.; Lopetegi, A.; Pérez, S.; Ruiz, M.; Tarriño, A. y Yusta, I. 2013: "La continuidad de la actividad pastoril durante la época romana en la zona de Argarbi (Sierra de Aralar)". Kobie XXXII: 5-28.

Mujika, J. A. y Edeso, J. M. 2012: Lehenengo nekazariabeltzainak Gipuzkoan Neolitikotik Burdin Aroral Los primeros agricultores y ganaderos en Gipuzkoa del Neolítico a la Edad del Hierro. Arkeologia 0.3, 382. Diputación Foral de Gipuzkoa. Donostia-San Sebastián.

Nájera, T.; Molina, F.; Jiménez-Brobeil, S. A.; SánchezRomero, M.; Al Oumaoui, I.; Aranda, G.; DelgadoHuertas, A. y Laffranchi, Z. 2010: "La población infantil de la Motilla del Azuer: un estudio bioarqueológico". Complutum 21: 69-102.

O'Leary, M. H. 1988: "Carbon isotopes in photosynthesis". BioScience 38: 325-336.

Post, D. M. 2002: "Using stable isotopes to estimate trophic position: models, methods, and assumptions". Ecology 83: 703-718.

Richards, M. P. y Hedges, R. E. M. 1999: "Stable isotope evidence for similarities in the types of marine foods used by Late Mesolithic humans at sites along the Atlantic Coast of Europe". Journal of Archaeological Science 26: 717-122.

Richards, M. P.; Price, T. D. y Koch, E. 2003: "The Mesolithic/ Neolithic transition in Denmark: new stable isotope data". Current Anthropology 44(2): 288-295.

Richards, M. P. y R. J. Schulting. 2006: "Touch not the fish: The Mesolithic-Neolithic change of diet and its significance". Antiquity 80 (308): 444-58.

Salazar-García, D. C. y Vizcaíno, A. 2011: “Análisis Isotópicos en Arqueología y Prehistoria. Estudio en curso de Cabezo Redondo y Cueva de las Lechuzas (Villena, Alicante)". Revista Villena: 169-176.

Sánchez, E. 2014: "La aplicación de isótopos estables en la Arqueología: el caso del $\delta^{18} \mathrm{O}$ en los restos óseos de una población de la Edad del Bronce de La Mancha: La Motilla del Azuer (Daimiel, Ciudad Real)". Arqueología y Territorio 11: 15-24.

Scheuer, L y Black, S. 2000: Developmental juvenile osteology. Academic Press. San Diego.

Schoeller, D. A. 1999: "Isotope fractionation: Why aren't we what we eat?". Journal of Archaeological Science 26: 667-673.

Schoeninger, M. J.; DeNiro, M. J. y Tauber, H. 1983: "Stable nitrogen isotope ratios of bone collagen re- 
flect marine and terrestrial components of prehistoric human diet". Science 220: 1381-1383.

Stloukal, M. y Hanáková, H. 1978: "Die Länge der Längsknochen altslawischer Bevölkerungen, unter besonderer Berücksichtigung von Wachstumsfragen". Homo 29: 53-69.

Tieszen, L. L. y Fagre, T. 1993: "Effect of diet quality and composition on the isotopic composition of pespiratory $\mathrm{CO} 2$, bone collagen, bioapatite, and soft tissues". En J. B. Lamber y G. Grupe (eds.): Prehistoric human bone. Archaeology at the molecular level. Springer-Verlag. Berlín: 121-155.

Ubelaker, D. H. 1989: "The estimation of age at death from immature human bone". En M. Y. Iscan (ed.): Age Markers in the Human Skeleton. Charles C. Thomas. Springfield: 55-70.

Van Klinken, G. J. 1999: "Bone collagen quality indicators for palaeodietary and radiocarbon measu- rements". Journal of Archaeological Science 26: 687-695.

Van Strydonck, M.; Boudini, M.; Ervynck, A.; Orvay, J. y Borms, H. 2005: "Spatial and temporal variation of dietary habits during the prehistory of the Balearic Islands as reflected by ${ }^{14} \mathrm{C}, \delta^{15} \mathrm{~N}$ and $\delta^{13} \mathrm{C}$ analyses on human and animal bones". Mayurqa 30: 523-541.

Warinner, C., Robles, N. y Tuross, N. 2013: "Maize, beans and the floral isotopic diversity of highland Oaxaca, Mexico". Journal of Archaeological Science 40: 868-873.

White, T. D. y Folkens, P. A. 2005: The human bone manual. Academic Press. San Diego.

Zavodny, E.; McClure, S. B.; Culleton, B. J.; Podrug, E. y Kennett, D. J. 2014: "Neolithic animal management practices and stable isotope studies in the Adriatic". Environmental Archaeology 19: 184-195. 